\title{
Quasi-Optical Power-Combining Arrays
}

\author{
David B. Rutledge, Zoya Basta Popović, Robert M. Weikle,II, \\ Moonil Kim, Kent A. Potter, Richard C. Compton*, Robert A. York* \\ Division of Engineering and Applied Science, California Institute of Technology \\ Pasadena, CA 91125, * School of Electrical Engineering \\ Cornell University, Ithaca, NY 14853
}

Abstract

Semiconductor devices have limited power handling capabilities at high frequencies, particularly at millimeter-wave frequencies. In this paper, we present a method for overcoming this problem by combining the outputs of several devices quasi-optically in a resonator cavity. This method has been applied to a number of solid-state devices, including Gunn diodes and MESFETs. The devices do not require an external locking signal because they lock to a mode of the resonator cavity. Effective radiated powers of 22 watts for a $4 \times 4$ array of Gunn diodes and 25 watts for a $10 \times 10$ array of MESFETs have been achieved.

\section{INTRODUCTION}

Vacuum tube sources, such as travelling-wave tubes and backward-wave oscillators are expensive, have short lifetimes, and require high-voltage power supplies when compared to their solid-state counterparts. However, the power generated by individual solid-state sources decreases rapidly at higher operating frequencies. The need for light-weight, reliable, watt-level sources (for millimeter-wave radar systems, for example) has thus resulted in a variety of power combining schemes for solid-state sources. The classical approach to power combining involves placing a number of sources in a waveguide cavity with tuning circuits for each source [1]. At higher frequencies, however, this approach becomes impractical and expensive as the components become lossy and difficult to build. Recently, it was suggested that the outputs of a large number of solid-state devices could be combined quasi-optically by placing the sources in a resonator cavity [2]. This approach avoids increased ohmic losses in the waveguide walls at higher frequencies and eliminates the need for individual tuning circuits for each device. By placing devices in a two-dimensional array and placing the array in a Fabry-Perot cavity, we obtain a power-combining grid whose configuration is reminiscent of a laser (Figure 1). A wide variety of grids have been previ- ously demonstrated, including detectors [3], phase-shifters [4], multipliers [5], and oscillators $[6,7,8]$. A grid loaded with active devices such as Gunn diodes or MESFET's produces a planar sheet with a reflection coefficient greater than unity. A resonator provides feedback that couples the devices together and permits the active sheet to be used as an oscillator. In this paper, we present two examples of power-combining grids, a 16-element Gunn diode array which utilizes patch antennas and a 100-element MESFET grid.

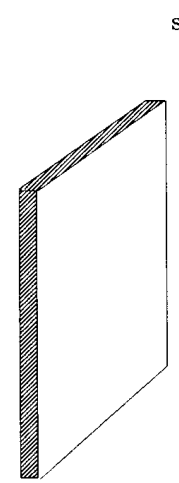

mirror substrate

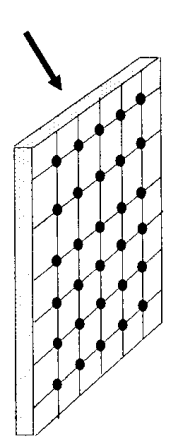

active grid dielectric slab

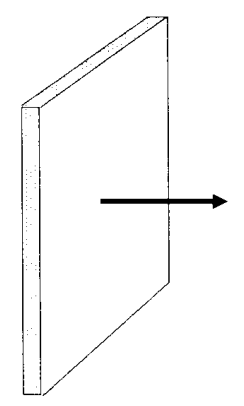

Figure 1. Quasi-optical power-combining array configuration. The active devices are placed on a substrate inside a Fabry-Perot cavity.

\section{Gunn Dione Power-Combining Array}

The Gunn diodes shown in Figure 2 are low-power packaged MA/COM devices. They are placed in a $4 \times 4$ array and feed patch antennas spaced half a free-space wavelength apart. The devices have individual bias lines, allowing a variety 
of biasing schemes. The threaded end of the diode package is screwed through the substrate and into the ground plane which also serves as a heat sink. This configuration is advantageous since Gunn diodes have low DC-to-RF efficiencies and most of the energy put into the devices must be removed as heat. The anode end-cap of the diode is left in contact with the patch antenna. DC bias is applied to the center of the non-radiating side of the patch.

The diode position under the patches and patch sizes for $10 \mathrm{GHz}$ oscillation were determined from investigations on single diode-patch elements. Dual patches were then fabricated to verify the concept of quasi-optical injection locking. With this experiment being successful, the array shown in Figure 2 was built and tested. Each diode was first biased one at a time to establish a common operating frequency. These individual biases were then applied simulataneously. Single-frequency operation was verified with a spectrum analyzer, and in-phase oscillation was verified by measuring

Top View

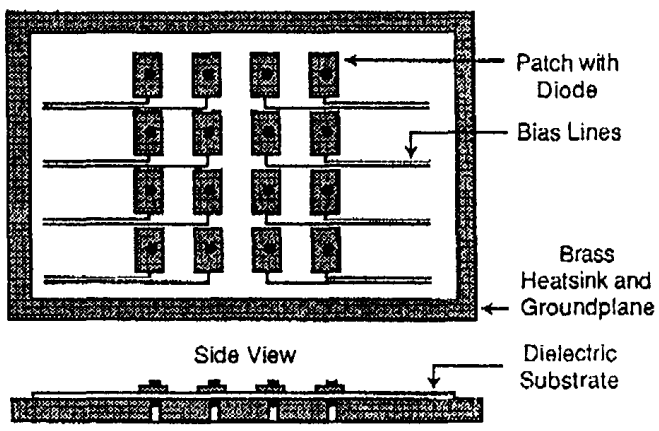

Figure 2. Schematic of a $4 \times 4$ array of Gunn diodes feeding microstrip patches. A dielectric slab is placed above the array to enhance locking.

the E-plane and H-plane patterns. Slight differences in the diode characteristics and diode placements made simultaneous inter-injection-locking a delicate operation and the addition of a dielectric slab above the array provided the necessary feedback to make locking routine.

The array was designed for $10 \mathrm{GHz}$ and oscillated at 9.6 $\mathrm{GHz}$. Sharp patterns in both the E-plane and H-plane corresponding to a directivity of $18 \mathrm{~dB}$ were obtained (Figure 3). A received power of $9.6 \mathrm{~mW}$ was obtained at a distance of 1.1 meters away using an $18 \mathrm{~dB}$ pyramidal horn. The total radiated power estimated from the measured patterns was $321 \mathrm{~mW}$, or about $20 \mathrm{~mW}$ per device. This agrees reasonably well with the $25 \mathrm{~mW}$ rating of the diodes. The effective radated power (ERP) of the array was 22 watts and the DC-to-RF conversion effieciency was around $0.7 \%$, which is typical for Gunn diodes.

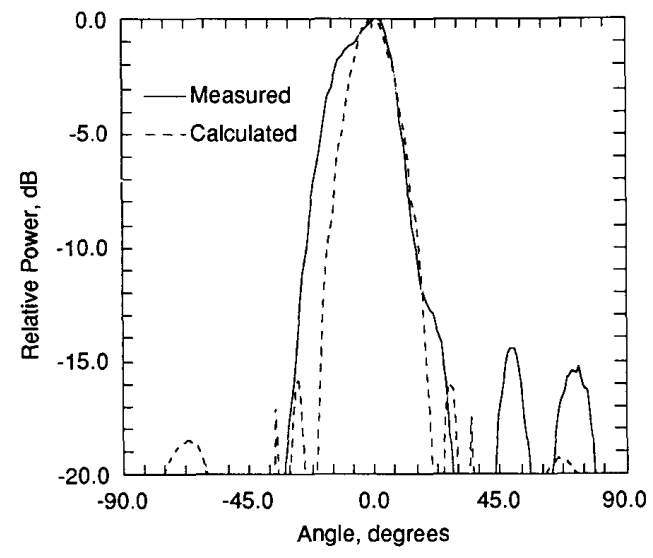

Figure 3, E-plane pattern for the active array of Fig. 2. The theoretical results are calculated by combining the pattern of a single patch with a $4 \times 4$ array factor.

\section{Planar MESFET Grid Oscillator}

Figure 4 shows the $10 \times 10$ array of MESFETs mounted on a $2.5 \mathrm{~mm}$ thick Duroid substrate $\left(\epsilon_{r}=10.2\right)$. The MESFETs share gate and drain bias lines with their neighbors and are $8 \mathrm{~mm}(0.133 \lambda)$ apart. A planar mirror is placed behind the grid to couple the devices together and also to serve as a tuning element. The measured far-field patterns are shown in Figure 5 and Figure 6 and give a directivity of $16 \mathrm{~dB}$. The ERP of the grid is measured to be 25 watts and the DC input power to the grid is 3 Watts. The power actually radiated from the grid was calculated to be $0.625 \mathrm{~W}$ which gives a DC-to-RF conversion efficiency of $20 \%$.

In order to predict the behavior of the grid, we need to determine the impedance presented to a device when it is placed in the grid. To derive a transmission-line model for the grid, we first use the symmetry of the grid to reduce it to an equivalent waveguide $[3,8]$. An EMF analysis is then performed for a MESFET placed inside the equivalent waveguide. This allows us to find the power spent in producing the fields and thus deduce the elements of the impedence matrix for the grid. The circuit that represents the grid is shown in Figure 7. The mirror and substrate are represented by a shunt reactance. A center-tapped transformer describes the coupling of the gate, drain, and source currents to the various waveguide modes. Simulation of the grid using this model was performed for various mirror positions. Figure 8 shows both the theoretical and measured mirror tuning curves. 


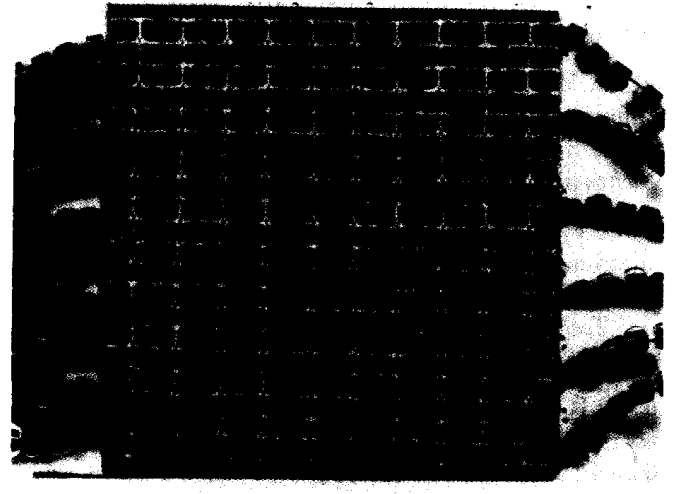

Figure 4. Photograph of the planar MESFET grid oscillator. The transistors are Fujitsu low-noise MESFET's (FSC11LF).

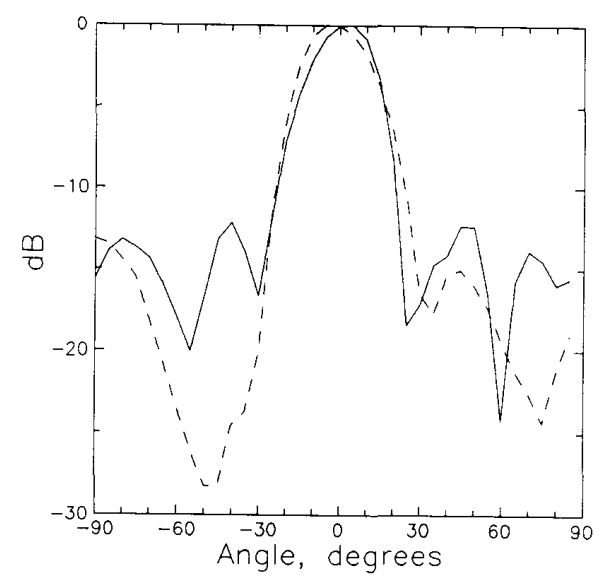

Figure 5. (-) E-plane and (- - ) H-plane antenna patterns of the MESFET grid oscillator.

The ability to lock an oscillator's output to some external signal has long been an important subject of study. Injection-locking stabilizes the oscillation frequency and allows the oscillator to perform various functions, such as generating, amplifying, and detecting an FM signal. External injection-locking of the planar MESFET grid was achieved by replacing the back reflector with a dielectric slab. The external signal was then injected from the back. A plot of locking bandwidth as a function of injected signal power is shown in Figure 9.

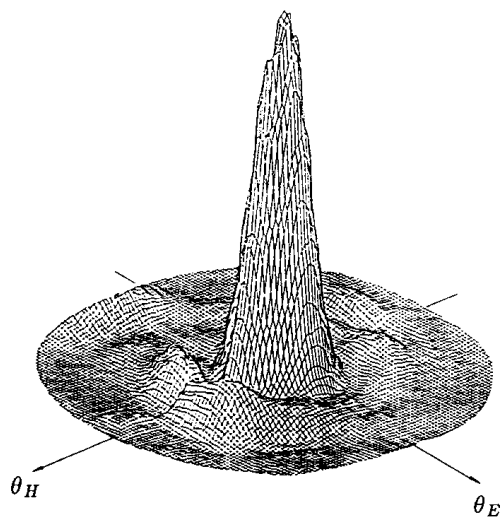

Figure 6. Far-field radiation pattern of the MESFET grid. The axes are given in terms of spherical coordinates by $\theta_{E}=\theta \sin \phi$ and $\theta_{H}=\theta \cos \phi$. The vertical scale is linear in power.

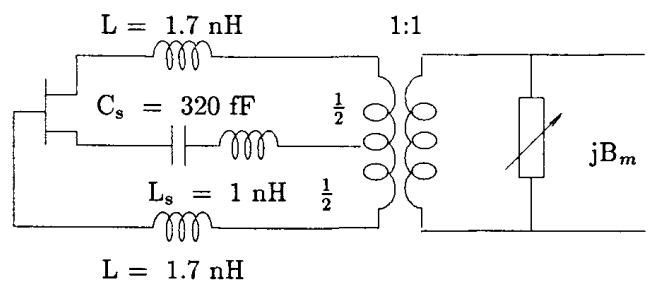

Figure 7. Transmission-line model for the planar MESFET grid. The values of the lumped elements are calculated from an EMF analysis of the grid.

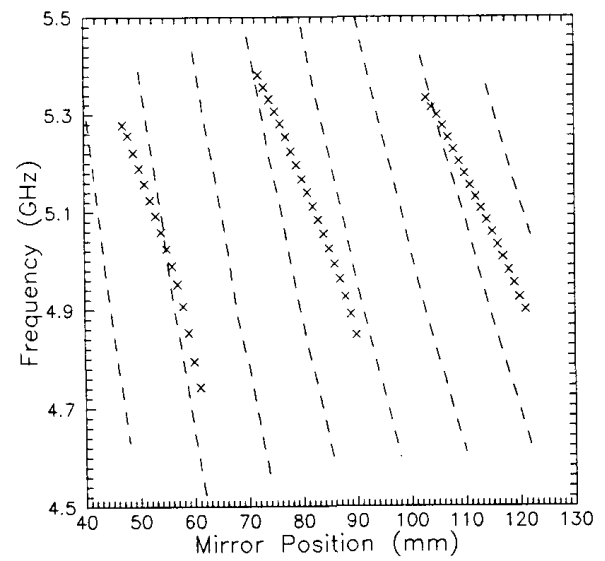

Figure 8. Theoretical (-) and measured $(x)$ mirror tuning curves for the planar MESFET grid. The theoretical curve was obtained by including the MESFET in the above model as a two-port with measured small-signal $s$-parameters. 


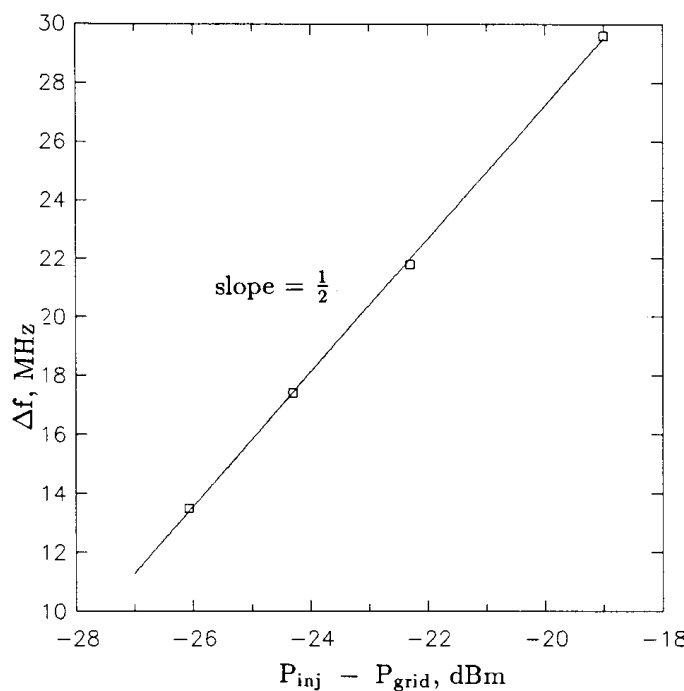

Figure 9. Injection-locking bandwidth for the MESFET grid as a function of $P_{i} / P_{o}$. A line with the theoretical slope of one-half is shown for comparison.

\section{CONClusions}

In this paper we have demonstrated two examples of quasioptical power-combining arrays that utilize both two- and three-terminal active devices. The array configuration is attractive because of its simplicity and its compatability with present-day IC fabrication techniques. The structure of these grids should be an advantage at higher frequencies where monolithic integration allows devices to be placed closer together and the grid would be many wavelengths across. This would result in a narrower beam and larger effective radiated power.

\section{ACKNOWLEDGEMENTS}

This research was supported by the Army Research Office, the Northrop Corporation, and General Electric.

\section{REFERENCES}

[1] K. Kurokawa, "The single-cavity multiple-device oscillator,"IEEE Trans. on Microwave Theory and Techniques, MTT-19, pp. 793-801, October 1971.

[2] J. W. Mink, "Quasi-Optical Power Combining of SolidState Millimeter-Wave Sources," IEEE Trans. on Microwave Theory and Techniques, MTT-94, pp. 273-279, February 1986.
[3] D. B. Rutledge, S. E. Schwarz,"Planar Multimode Detector Arrays for Infrared and Millimeter-Wave Applications," IEEE Journal of Quantum Electronics, QE-17, No.S, pp. 407-414, March 1981.

[4] W. W. Lam, C. F. Jou, H. Z. Chen, K. S. Stolt, N. S. Luhmann, D. B. Rutledge, "Millimeter-Wave Diode-Grid Phase Shifters," IEEE Trans. on Microwave Theory and Techniques, MTT-36, No. 5, pp. 902-907, May 1988.

[5] C. F. Jou, W. W. Lam, H. Z. Chen, K. S. Stolt, N. S. Luhmann, D. B. Rutledge, "Millimeter-Wave Diode-Grid Frequency Doubler" IEEE Trans. on Microwave Thoery and Techniques," MTT-36, No. 11, pp. 1507-1514, Novermber 1988 .

[6] Z. B. Popović, M. Kim, D. B. Rutledge, "Grid Oscillators," International Journal of Infrared and Millimeter Waves, 9, No. 7, pp. 647-654, July 1988.

[7] M. Hieda, M. Nakayama, K. Mizuno, T. Ajikata, D. Rutledge, "Quasi-Optical Resonator for Millimeter and Submillimeter Wave Solid-State Sources," 13th International Conference on Infrared and Millimeter Waves, Conference Digest, pp. 55-56, December 1988.

[8] Z. B. Popović, R. M. Weikle, M. Kim, K. A. Potter, D. B. Rutledge,"Bar-Grid Oscillators," to be published in IEEE Trans. on Microwave Theory and Techniques. 